\title{
Acute myocardial infarction due to left main coronary artery disease in men and women: does ST-segment elevation matter?
}

\author{
Marcin Sadowski ${ }^{1}$, Wojciech Gutkowski ${ }^{1}$, Grzegorz Raczyński ${ }^{1}$, Agnieszka Janion-Sadowska², \\ Marek Gierlotka³ ${ }^{3}$ Lech Poloński
}

\author{
${ }^{1}$ Department of Interventional Cardiology, Świętokrzyskie Cardiology Center, Kielce, \\ Poland \\ ${ }^{2}$ Intensive Cardiac Care Unit, Świętokrzyskie Cardiology Center, Klelce, Poland \\ ${ }^{3} 3^{\text {rd }}$ Department of Cardiology, Silesian Center for Heart Diseases, Zabrze, Poland
}

Submitted: 31 July 2013

Accepted: 23 January 2014

Arch Med Sci 2015; 11, 6: 1197-1204

DOI: 10.5114/aoms.2015.56345

Copyright $\odot 2015$ Termedia \& Banach

\author{
Corresponding author: \\ Marcin Sadowski MD, PhD \\ Świętokrzyskie Cardiology \\ Center \\ 45 Grunwaldzka St \\ 25-736 Kielce, Poland \\ Phone: +48413671580 \\ E-mail: emsad@o2.pl
}

\begin{abstract}
Introduction: Gender-specific issues regarding ST-segment elevation (STEMI) and non-ST-segment elevation myocardial infarction (NSTEMI) due to unprotected left main coronary artery (ULMCA) disease were not sufficiently studied. We assessed the value of STEMI/NSTEMI initial classification on the management of men and women with acute MI due to critical stenosis or occlusion of the ULMCA.

Material and methods: The study group consisted of 643 consecutive patients with acute MI with the ULMCA as the infarct-related artery. Data derive from an ongoing, nationwide, multicenter, prospective, observational registry.

Results: Isolated ULMCA disease was more frequent in women and multivessel disease was more frequent in men in the NSTEMI group. The incidence of cardiogenic shock or pulmonary edema and cardiac arrest was higher in the STEMI group. Totally occluded ULMCA was more frequent in the STEMI group. Although the majority of patients underwent percutaneous coronary intervention $(\mathrm{PCI})$, it was less frequently used in NSTEMI women and NSTEMI men. Although in-hospital and long-term mortality rates were higher in the STEMI group, there were no gender-related differences within groups. The initial ST-segment elevation was an independent predictor of in-hospital $(\mathrm{OR}=2.37,95 \% \mathrm{Cl}: 1.14-4.91, p=0.02)$ and 12 -month $(\mathrm{OR}=1.52$, $95 \% \mathrm{Cl}: 1.01-2.27, p=0.045)$ mortality.

Conclusions: There were no gender-related differences in the management within the STEMI or NSTEMI group. Although acute myocardial infarction due to ULMCA disease is associated with high mortality in both genders, STEMI was a negative prognostic factor of in-hospital and 12-month mortality. Despite poor baseline characteristics and clinical presentation in women, female gender itself did not influence mortality.
\end{abstract}

Key words: myocardial infarction, ST-segment, left main, gender, women.

\section{Introduction}

The unprotected left main coronary artery (ULMCA) supplies up to 75-100\% of left ventricular mass depending on the dominance type [1]. For that reason acute non-ST-segment elevation (NSTEMI) or ST-seg- 
ment elevation (STEMI) myocardial infarction due to critical stenosis or abrupt occlusion of the ULMCA is a catastrophic situation with a very high in-hospital and long-term mortality [2]. Many cases are never reported because of pre-hospital death. Although coronary artery bypass grafting (CABG) remains a class I recommendation for $L M$ revascularization in European and American guidelines, percutaneous coronary intervention $(\mathrm{PCl})$ is becoming an attractive option in patients with acute myocardial infarction and ULMCA as an infarct-related artery, especially when in cardiogenic shock [3, 4]. Advances in devices and adjunctive pharmacotherapy make $\mathrm{PCl}$ of the ULMCA feasible and with at least non-inferior results to CABG [5-7]. Although electrocardiography is not a highly specific method for the diagnosis of myocardial infarction due to ULMCA disease, the primary results of our registry suggest that STEMI (vs. NSTEMI) remains an independent predictor of in-hospital and 12-month mortality [8]. Several lines of evidence indicate that not all patients with severe ULMCA disease develop ECG changes before a hemodynamic collapse. In those who present with ST-segment abnormalities an aVR lead is one of high specificity and sensitivity for ULMCA disease, especially when ST-elevation is higher than in the V1 lead, which correlates with mortality and hemodynamic deterioration $[9,10]$. Mahajan et al. reported that differences in ST-segment deviations in the V 1 and $\mathrm{V} 6$ leads are even more specific for predicting ULMCA disease than the aVR lead itself [11].

An initial diagnosis of STEMI or NSTEMI is crucial for determining the patients' flow and further management. Gender-specific issues were precisely analyzed in our country both for STEMI and NSTEMI patients $[12,13]$, but data on the patients' management in acute $\mathrm{MI}$ and the ULMCA as an infarct-related artery are scarce. The aim of our study was to assess whether the initial classification of STEMI vs. NSTEMI and the classification-related therapeutic approach influence mortality in men and women with acute myocardial infarction due to critical stenosis or occlusion of the ULMCA.

\section{Material and methods}

The principles of our registry (PL-ACS) have been reported elsewhere [14]. Briefly, this is an ongoing, nationwide, multicenter, prospective, observational mandatory registry of all consecutive acute coronary syndrome (ACS) cases in Poland. So far, there are over 500000 cases recorded. The study group consisted of 643 consecutive patients hospitalized during three years (from October 2003 to August 2006) with acute MI with ULMCA as the infarct-related artery (IRA) defined as stenosis over
$50 \%$ or total occlusion. Cases with IRA other than ULMCA but with significant ULMCA disease were excluded from the analysis. Basic clinical characteristics, treatment strategy and prognosis for the entire population with ULMCA-related MI were reported previously [8]. Subjects were divided into STEMI and NSTEMI groups and into females and males within each group. Data analyzed included information from patients' history, coronary risk factor profile, clinical presentation, therapeutic approach and adjunctive treatment. The primary end-points were in-hospital, 30-day, 6-month and 12-month mortality. Mortality data were obtained for all subjects included from the governments' official mortality records.

\section{Statistical analysis}

Variables were expressed as mean \pm standard deviation, counts and percentages or median and interquartile ranges as appropriate. The significance between groups was tested using Student's $t$-test, the Mann-Whitney $U$ test or Kruskal-Wallis ANOVA test depending on normality as well as homogeneity of variances tested by the $F$ test. Categorical variables were tested by the $\chi^{2}$ test. Follow-up mortality was analyzed using the Kaplan-Meier method for multiple-group comparisons. A two-sided $p$-value $\leq 0.05$ was considered significant. For all calculations, Statistica 7.1 software (StatSoft, Inc., Tulsa, OK, USA) was used.

\section{Results}

ST-segment elevation myocardial infarction patients were on average 4 years younger than NSTEMI patients $(p<0.0001)$ and less frequently developed arterial hypertension (60.0\% vs. $73.4 \%$; $p<0.0003$ ). Women with STEMI more often were smokers than women with NSTEMI, whereas NSTEMI men more often than STEMI men had a previous myocardial infarction (Table I). While there was no difference between genders in the extent of the disease in the STEMI group, an isolated ULMCA disease was more frequent in women and multivessel disease was more frequent in men in the NSTEMI group. The incidence of cardiogenic shock or pulmonary edema, activity of myocardial isoenzyme of creatine phosphokinase, cardiac arrest and larger extent of the coronary artery disease were higher in the STEMI group (Table II).

There were no differences in medication between STEMI men and women. The NSTEMI women slightly less frequently received nitrates as compared with NSTEMI men (47.9\% vs. $60.3 \%$; $p<0.038)$. ST-segment elevation myocardial infarction patients, both men and women, as compared with NSTEMI patients significantly less frequently received low molecular weight heparins, 
$\beta$-blockers, calcium channel antagonists, angiotensin-converting enzyme inhibitors and statins (data not shown).

Both males and females in the STEMI group presented more frequently with totally occluded ULMCA. Although the majority of patients underwent $\mathrm{PCl}$, it was less frequently used in NSTEMI women and NSTEMI men. Similarly, the use of glycoprotein IIb/IIla inhibitors in the NSTEMI subgroups was lower (Table III).

The complication rate was very low. Only repeat NSTEMI and unstable angina were more frequent in the NSTEMI group, both in females and in males (Table IV). Treatment outcomes were similar for both genders, with a high rate of post-procedural TIMI 3 flow (Table IV). In-hospital and long-term mortality rates were higher in the STEMI vs. NSTEMI group (in-hospital: $27.2 \%$ vs. $10.4 \%, p<0.0001$; 12 -month: $38.4 \%$ vs. $24.6 \%$, $p<0.0001$ ) [8]. There were no differences in mortality between genders within the STEMI and NSTEMI groups in all patients and subgroups treated medically and invasively. However, in patients who underwent a conservative strategy and in those treated invasively significant differences were noticeable in favor of NSTEMI (Table V). As we have previously reported [8], together with cardiogenic shock, pulmonary edema and advanced age, initial ST-elevation on ECG was an independent predictor of in-hospital $(\mathrm{OR}=2.37,95 \% \mathrm{Cl}$ : 1.14-4.91, $p=0.02)$ and 12-month $(\mathrm{OR}=1.52$, $95 \% \mathrm{Cl}: 1.01-2.27, p=0.045)$ mortality.

\section{Discussion}

Despite the increasing number of reports on primary angioplasty in the unprotected left main coronary artery, CABG remains the preferred treatment option for patients with this localization of the lesion. Both American and European cardiac societies recommend the quickest possible recanalization of acutely occluded vessels in patients with acute $\mathrm{MI}$ (level IA recommendation). It is interesting that most clinical trials evaluating outcomes of left main coronary artery angioplasty as compared with other therapies exclude patients with acute coronary syndromes. Only large registries such as GRACE [15], the meta-analysis by Lee et al. [16] and the present study have taken into account the physician's approach to a serious medical condition such as acute coronary syndrome due to significant left main coronary artery stenosis. Even in one of the recent large randomized studies, the SYNTAX trial comparing coronary angioplasty and coronary artery bypass grafting in patients with triple vessel disease or left main coronary artery disease, myocardial infarction was an exclusion criterion [17]. This may cause unnecessary hesitation when an interventional cardiolo-

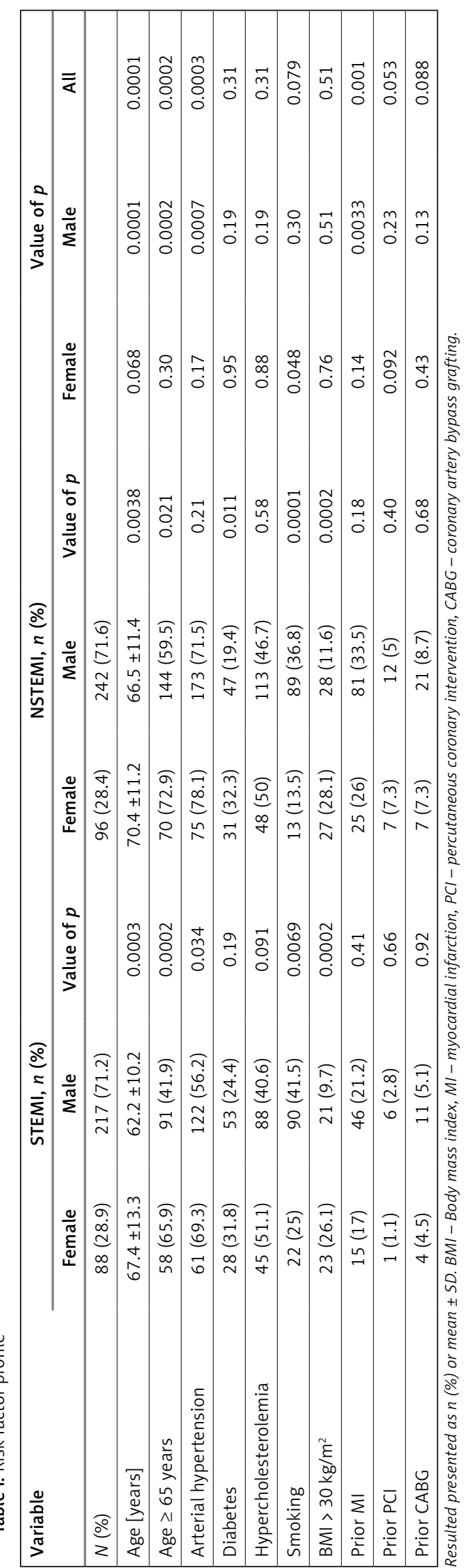




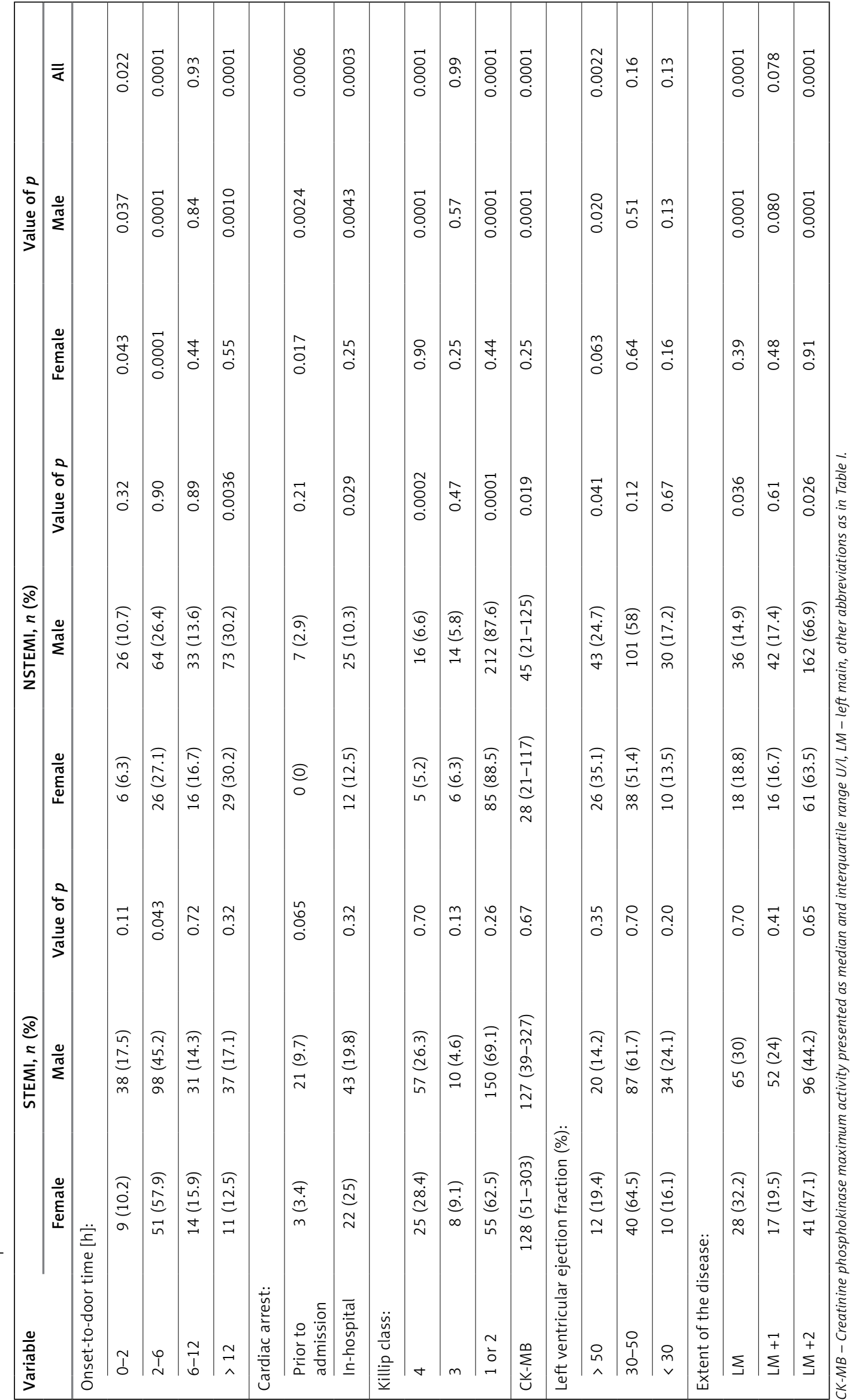




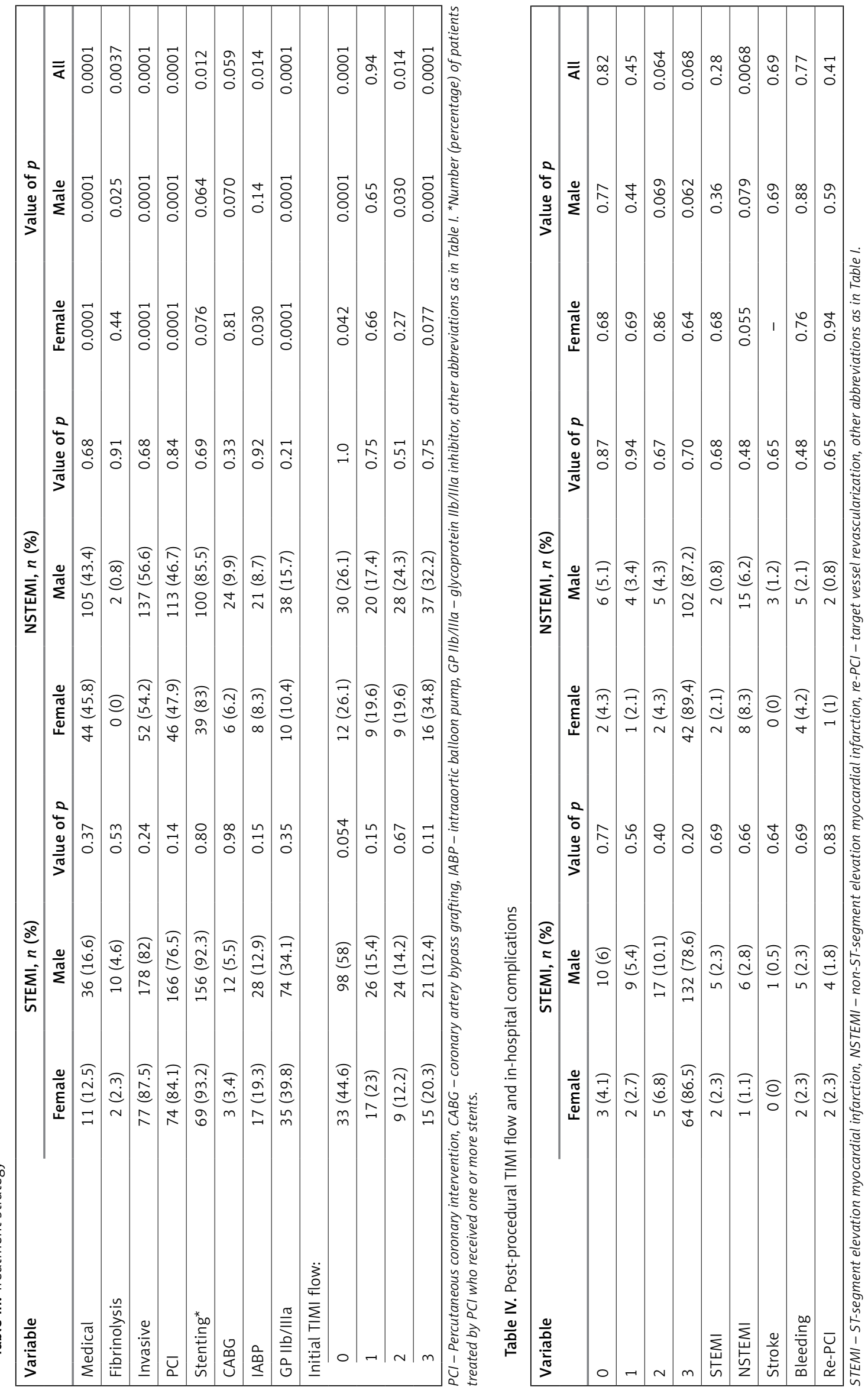




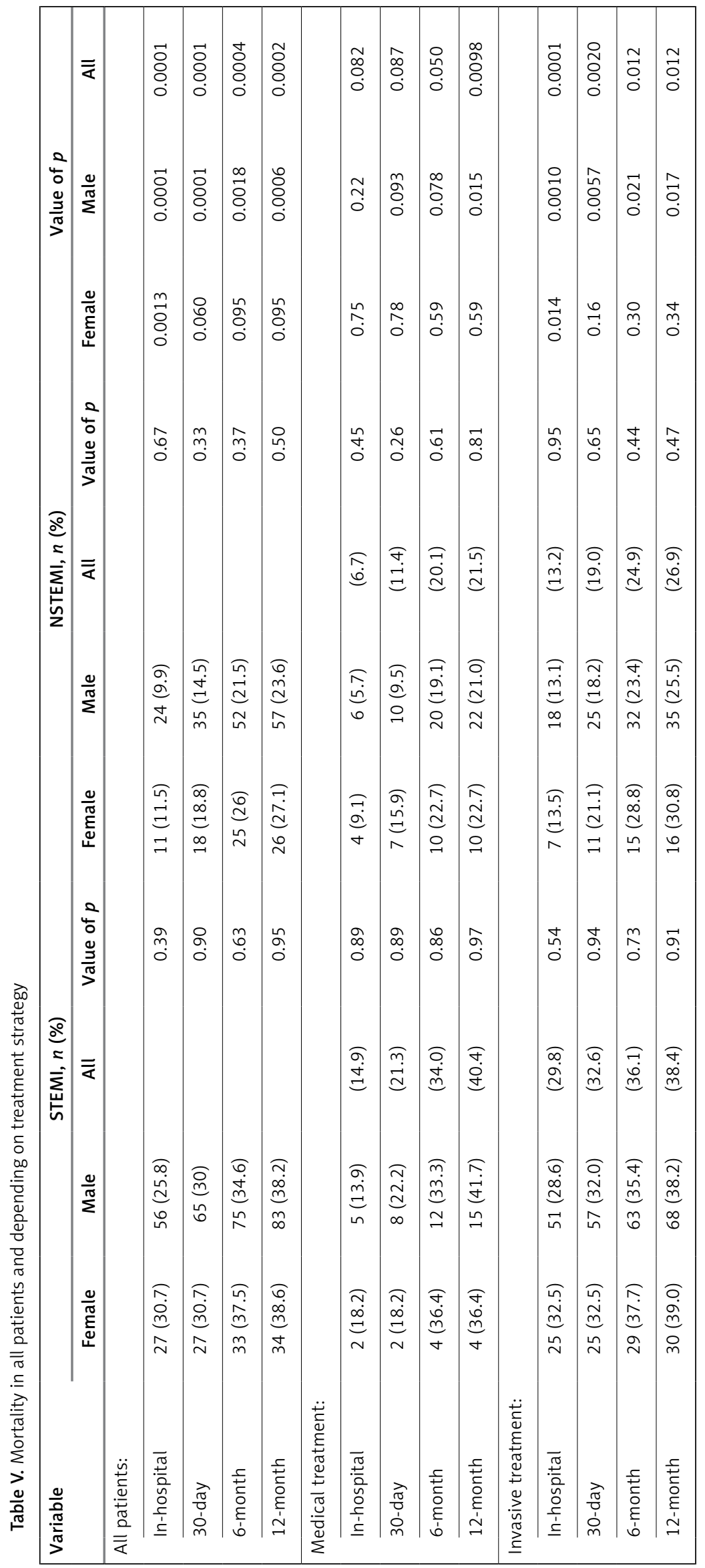


gist sees such a patient, being aware of increased risk for the patient on the one hand and bearing in mind mandatory guidelines on the other. Leaving the vessel occluded in patients evolving with cardiogenic shock is unlikely. However, it is a different situation when the patient is in a satisfactory clinical condition despite critical LM stenosis and manifestations of acute coronary syndrome. The commonly used scores such as EuroSCORE and Parsonnet evaluate cardiac operative risk and encourage interventional cardiologists to perform $\mathrm{PCl}$ - the higher the operative risk, the easier is the decision to perform coronary angioplasty. Other scores used in multicenter studies of acute coronary syndromes such as GRACE and the SYNTAX Score lead to similar decisions $[15,18$, 19]. The higher the score, the higher is the operative risk and the more difficult it is to make a decision, both for interventional cardiologists and cardiac surgeons. Unfortunately, none of the scores include ST-segment deviation. Although the definition of myocardial infarction has been extended to include all patients who present with myocardial necrosis biomarkers, STEMI most frequently develops in subjects with total vessel occlusion with TIMI flow grade $0 / 1$. The majority of these patients also have such complications as cardiogenic shock and/or cardiac arrest. The utility of initial classification of STEMI vs. NSTEMI in the context of ULMCA disease has been thoroughly studied in terms of both clinical outcomes [20] and identifying the culprit lesion [21]. In our study it was clearly demonstrated that patients presenting with the STEMI pattern have shorter onset-to-door times and are more frequently treated invasively (both $\mathrm{PCl}$ and/or $(A B G)$. However, treatment allocation ( $P C l$ vs. $C A B G)$ is often based on the TIMI flow grade, and STEMI patients have a better chance for immediate revascularization regardless of their condition [22]. Furthermore, STEMI is associated with isolated LM stenosis in contrast to NSTEMI, which occurs in patients with multivessel disease. Another interesting fact about the present study is age. Patients with STEMI were younger than those with NSTEMI. In general, STEMI patients had fewer comorbidities than NSTEMI subjects. The reasons for this difference can be related to factors initiating the development of collaterals, allowing quick opening of coronary collaterals and protection of blood flow beyond the occluded segment. Available registries reflect this approach. Most STEMI patients were treated by means of coronary angioplasty. Montalescot et al. analyzed data from the GRACE registry and made interesting observations. During the data collection period from 2000 to 2007 there was a shift from coronary artery bypass grafting towards coronary angioplasty performed in patients with acute coronary syndrome caused by critical LM stenosis (in 2004/2005) [23].
Most investigators emphasize the fact of quick and total restoration of blood flow after LM stenting. Also in the present study TIMI flow grade 2 and 3 was achieved in over $90 \%$ of patients undergoing $\mathrm{PCl}$, which was performed in almost $80 \%$ of STEMI and in $47 \%$ of NSTEMI patients, in contrast to CABG performed in $3.9 \%$ and $7.4 \%$ of patients, respectively. Time to flow restoration depended largely on time to admission. In our study, STEMI patients were admitted earlier than their NSTEMI counterparts. The selection process for CABG is different. Analysis of the GRACE registry showed that $\mathrm{PCl}$ was performed on the day of admission and the time to recanalization did not exceed $24 \mathrm{~h}$, whereas the mean selection time for CABG was 4.5 days.

Despite well-documented female sex-related discrepancies in the presentation, management, clinical course and outcomes in patients with STEMI or NSTEMI in the general population [12, 13], in the elderly [24] and in the young [25], in the present study we did not find any significant male-favoring difference. This is a surprising finding, leading to the conclusion that the deleterious impact of the ULMCA-related myocardial infarction on mortality is the greatest of all known risk factors. Whereas gender-related differences did not matter in the present study, we were able to demonstrate that patients with STEMI had greater mortality regardless of the modality of treatment. Initial classification of STEMI vs. NSTEMI allowed us to identify patients at high risk.

Our database is not free of flaws typical for other registries. Although data collection and case reporting are mandatory, we had no impact on data integrity. Moreover, the discrimination of ULMCA stenosis severity and each therapeutic decision were operator-dependent. Our registry did not allow us to collect data on the lesion location and complexity (i.e. ostial vs. bifurcation) or stenting strategy. Neither vascular access site selection nor any sophisticated parameters were considered, although they are also known to impact the mortality, as it has been recently reported $[26,27]$. Mortality data, although obtained for all cases included, did not distinguish between cardiovascular and all-cause mortality. No follow-up data regarding post-discharge patient compliance or repeat hospitalizations and revascularization are available. Therefore, extrapolation of our results must be done with caution.

In conclusion, there were no gender-related differences in the management within the STEMI or NSTEMI group. Although acute myocardial infarction due to ULMCA critical stenosis or occlusion is associated with high mortality in both genders, STEMI was a negative prognostic factor of in-hospital and 12-month mortality. In the STEMI group, mortality was greater regardless of treatment 
strategy. Despite poor baseline characteristics and clinical presentation in women, female gender itself did not influence mortality.

\section{Conflict of interest}

The authors declare no conflict of interest.

\section{References}

1. Kalbfleisch H, Hort W. Quantitative study on the size of coronary artery supplying areas postmortem. Am Heart J 1977; 94: 183-8

2. Atie J, Burgada P, Burgada J, et al. Clinical presentation and prognosis of left main coronary artery disease in the 1980s. Eur Heart J 1991; 12: 495-502.

3. Puricel S, Adorjan P, Oberhänsli $M$, et al. Clinical outcomes after $\mathrm{PCl}$ for acute coronary syndrome in unprotected left main coronary artery disease: insights from the Swiss Acute Left Main Coronary Vessel Percutaneous management (SALVage) study. Eurolntervention 2011; 7: 697-704.

4. Lee MS, Bokhoor P, Park SJ, et al. Unprotected left main coronary disease and ST-segment elevation myocardial infarction: a contemporary review and argument for percutaneous coronary intervention. JACC CardiovasC Interv 2010; 3: 791-5.

5. Kandzari DE, Colombo A, Park SJ, et al. Revascularization for unprotected left main disease: evolution of the evidence basis toredefine treatment standards. J Am Coll Cardiol 2009; 54: 1576-88.

6. Seung KB, Park DW, Kim YH. Stents versus coronary-artery bypass grafting for left main coronary artery disease. N Engl J Med 2008; 358: 1781-92.

7. Serruys PW, Morice MC, Kappentein AP, et al. Percutaneous coronary interventions versus coronary-artery bypass grafting for severe coronary artery disease. N Engl Med 2009; 360: 961-72

8. Sadowski M, Gutkowski W, Janion-Sadowska A, et al. Acute myocardial infarction due to left main coronary artery disease: a large multicenter national registry. Cardiol J 2013; 20: 190-6.

9. Yamaji $\mathrm{H}$, Iwasaki K, Kusachi S, et al. Prediction of acute left main coronary artery obstruction by 12 -lead electrocardiography. ST segment elevation in lead aVR with less ST segment elevation in lead V1. J Am Coll Cardiol 2001; 38: 1348-54.

10. Barrabes JA, Figueras J, Moure C, et al. Prognostic value of lead aVR in patients with first non ST segment elevation acute myocardial infarction. Circulation 2003; 108: 814-9.

11. Mahajan N, Hollander G, Thekkoott D, et al. Prediction of left main coronary artery obstruction by 12 -lead electrocardiography: ST segment deviation in lead V6 greater than or equal to ST segment deviation in lead V1. Ann Noninvasive Electrocardiol 2006; 11: 102-12.

12. Sadowski M, Gasior M, Gierlotka M, Janion M, Poloński L. Clinical characteristics of Polish women with ST-segment elevation myocardial infarction. Kardiol Pol 2010; 68: 627-34.

13. Janion-Sadowska A, Sielski J, Gierlotka M, NowalanyKozielska E, Janion M, Poloński L. Gender-related differences in clinical course, therapeutic approach and prognosis in patients with non-ST segment elevation myocardial infarction. Kardiol Pol 2011; 69: 784-92.

14. Poloński L, Gąsior M, Gierlotka M, et al. Polish Registry of Acute Coronary Syndromes (PL-ACS). Characteristics, treatments and outcomes of patients with acute coronary syndromes in Poland. Kardiol Pol 2007; 65: 861-72.

15. Fox KAA, Anderson Jr FA, Dabbous OH, et al. Intervention in acute coronary syndromes: do patients undergo intervention on the basis of their risk characteristics? The Global Registry of Acute Coronary Events (GRACE). Heart 2007; 93: 177-82.

16. Lee MS, Sillano D, Latib A, et al. Multicenter international registry of unprotect left main coronary artery percutaneus coronary intervention with drug-eluting stents in patients with myocardial infarction. Cath Cardiovasc Interv 2009; 73: 15-20.

17. Serruys PW, Morice MC, Kappetein AP, et al. Percutaneus coronary intervention versus coronary-artery bypass grafting for severe coronary artery disease. N Engl J Med 2009; 360: 961-72.

18. Chieffo A, Stankowic G, Bonizzoni E, et al. Early and mid-term results of drug-eluting stent implantation in unprotect left main. Circulation 2005; 111: 791-5.

19. Serruys PW, Morice MC, Kappetein AP, et al. Percutaneus coronary intervention versus coronary-artery bypass grafting for severe coronary artery disease. N Engl J Med 2009; 360: 961-72.

20. Alherbish A, Westerhout CM, Fu Y, et al. The forgotten lead: does aVR ST-deviation add insight into the outcomes of ST-elevation myocardial infarction patients? Am Heart J 2013; 166: 333-9.

21. Kühl JT, Berg RM. Utility of lead aVR for identifying the culprit lesion in acute myocardial infarction. Ann Noninv Electrocardiol 2009; 14: 219-25.

22. Grundeken MJ, Vis MM, Beijk MA, et al. Clinical outcomes after percutaneous or surgical revascularisation of unprotected left main coronary artery-related acute myocardial infarction: a single-centre experience. Heart 2013; 99: 690-9.

23. Montalescot G, Brieger D, Eagle KA, et al. Unprotected left main revascularization in patients with acute coronary syndromes. Eur Heart J 2009; 30: 2308-17.

24. Sielski J, Janion-Sadowska A, Sadowski M, et al. Differences in presentation, treatment, and prognosis in elderly patients with non-ST-segment elevation myocardial infarction. Pol Arch Med Wewn 2012; 122: 253-61.

25. Sadowski M, Janion-Sadowska A, Gąsior M, Gierlotka M, Janion M, Poloński L. Higher mortality in women after ST-segment elevation myocardial infarction in very young patients. Arch Med Sci 2013; 9: 427-33.

26. Komócsi A, Aradi D, Kehl D, et al. Meta-analysis of randomized trials on access site selection for percutaneous coronary intervention in ST-segment elevation myocardial infarction. Arch Med Sci 2014; 10: 203-12.

27. Jeżewski T, Peruga JZ, Kasprzak JD, et al. In-hospital daily insulin dose predicts long-term adverse outcome in patients with diabetes with ST-elevation myocardial infarction treated with successful primary percutaneous angioplasty. Arch Med Sci 2014; 10: 913-9. 